MS13-O2 Challenges at low resolution: crystal structure of the yeast VPS34 complex II.

Nicolas Soler ${ }^{1}$, Ksenia Rostislavleva ${ }^{1}$, Yohei Ohashi ${ }^{1}$, Lufei Zhang $^{1}$, Els Pardon ${ }^{2,3}$, John E. Burke ${ }^{1}$, Glenn R. Masson ${ }^{1}$, Chris Johnson ${ }^{1}$, Jan Steyaert ${ }^{2,3}$, Nicholas T. Ktistakis ${ }^{4}$, Roger L. Williams $^{1}$

1. MRC Laboratory of Molecular Biology, Cambridge, UK

2. Structural Biology Research Center, VIB, Brussels, Belgium.

3. Structural Biology Brussels, Vrije Universiteit Brussel, Brussels, Belgium.

4. The Babraham Institute, Cambridge, UK

email: nicolas@nicolassoler.eu

Vps34 is a phosphatidylinositol 3-kinase that regulates intracellular vesicle trafficking in endocytic sorting, cytokinesis and autophagy. In cells, the enzyme occurs in two large protein complexes known as complex I and complex II. The full-length 385 kDa yeast endosomal complex II, which consists of Vps34, Vps15, Vps30 and Vps38 could be crystallized using a nanobody that binds to Vps34 and the $4.4 \AA$ structure was subsequently solved. The Y-shaped complex shows Vps34 and Vps15 closely engaging in one arm and $\mathrm{Vps} 30$ and $\mathrm{Vps} 38$ form the other arm. Hydrogen-Deuterium Exchange Mass Spectrometry (HDX-MS) revealed dynamics of the complex and showed that conformational changes accompany membrane binding and lipid kinase activity. HDX-MS identified a loop in Vps30 that controls the activity on the enzyme on lipid membranes. Determining the structure at this resolution required careful MAD data collection for a tantalum cluster to give excellent phases, which revealed the general shape of the complex. Most of the structure had to be built de novo into this electron density map. However, this process was assisted by first segmenting the structure into elements then employing an automated script using secondary structure profiling results (HHPRED) to place pieces of known structure into the local density. The model was then completed and finalised with manual model building.

Keywords: Vps34, complex II, autophagy, endosomal sorting, nanobody, low resolution.

\section{MS13-O3 X-Ray Crystal Structure of a Chromatosome}

Curt A. Davey ${ }^{1}$, Zenita Adhireksan ${ }^{1}$, Qiuye Bao ${ }^{1}$, Sivaraman Padavattan $^{1}$

1. School of Biological Sciences, Nanyang Technological University, 60 Nanyang Drive, Singapore 637551

email: davey@ntu.edu.sg

Linker histones are essential proteins in higher eukaryotes that promote chromatin compaction through binding to the nucleosome. Condensing nucleosomes into highly compact states is required for gene regulation, maintaining genomic integrity and generating mitotic chromosomes for cell division. Nonetheless, the biological relevance of different types of condensed nucleosomal structures observed in vitro is controversial, and we lack a unified understanding of how linker histones bind to nucleosomes and promote compaction in the cell.

The first atomic model of the nucleosome core (particle) was published in 1997 [1], paving the way for the structural solution of a great variety of nucleosome core constructs composed of variant/modified histones and different DNA sequences [2,3]. This has consequently revolutionized our understanding of nucleosome activity, and yet nearly two decades later we are still lacking an atomic level understanding of how linker histones interact with nucleosomes.

As with the nucleosome core particle, the challenge in solving the first structure of a chromatosome-the nominal assembly of a nucleosome and linker histone-has been in the discovery of constructs that yield well diffracting crystals. To overcome this obstacle, we developed an approach that promotes ordered lattice formation, which appears to have general utility for structural studies of nucleosomal assemblies. Here we will describe the $2.7 \AA$ resolution X-ray structure of a chromatosome composed of a full length $\mathrm{H} 1$ variant. The structure reveals a hitherto unknown mode for linker histone-induced condensing of nucleosomes, which rationalizes in vivo data and provides a simple model behind chromatin compaction.

[1] K. Luger, A.W. Mäder, R.K. Richmond, D.F. Sargent \& T.J. Richmond. 1997. Crystal structure of the nucleosome core particle at $2.8 \AA$ resolution. Nature. 389: 251-60.

[2] S. Tan \& C.A. Davey. 2011. Nucleosome structural studies. Curr. Opin. Struct. Biol. 21: 128-136.

[3] E.Y.D. Chua*, D. Vasudevan*, G.E. Davey, B. Wu \& C.A. Davey. 2012. The mechanics behind DNA sequence-dependent properties of the nucleosome. Nucleic Acids Res. 40: 6338-6352.

Funding: Ministry of Education Academic Research Fund Tier 2 (grant MOE2015-T2-2-089); Ministry of Education Academic Research Fund Tier 3 Programme (grant MOE2012-T3-1-001).

Keywords: Nucleosome, Linker histone, Chromatin, Chromatosome, DNA compaction 